\title{
数字高程模型在群落内物种共存研究中的应用 ——以神农架米心水青冈-曼青冈群落的地形模型建立为例
}

\author{
张 烅1,2 熊高明 1 陈志刚 ${ }^{1}$ 樊大勇 ${ }^{1}$ 谢宗强 ${ }^{*}{ }^{*}$ \\ （1 中国科学院植物研究所植被数量生态学重点实验室, 北京 100093）（2 中国科学院研究生院, 北京 100039)
}

摘 要 地形是环境异质性产生的原因之一。受技术条件的限制, 以往研究中地形因子只能作为背景因子介入, 无法分析地形的细微变化对植物分布格局的影响。该文将数字高程模型引入群落内物种多样性研究, 以神农架米 心水青冈 (Fagus engleriana)- 曼青冈 (Cyclobalanopsis oxyodon) 群落固定样地的地形分析为例, 介绍 DEM 的建立及地 形因素的提取。摸拟 $0.96 \mathrm{hm}^{2}$ 样地的地形变化, 发现样地总坡向为西偏北 $30^{\circ}$, 总坡度为 $40.01^{\circ}$, 最大坡度为 $74.6^{\circ}$, 最小坡度为 $18.2^{\circ}$, DEM 的精度为 $0.4 \mathrm{~m}$ 。选择群落中 24 种植物与坡度、坡向及坡位等地形因素进行相关分析, 发 现不同的种对地形变化的反应不同。该次研究中, DEM 量化了地形数据, 使之可以参与到生物多样性研究中, 为在 连续面上分析地形因素对群落内植物分布的影响提供了极大的便利。DEM 在小尺度中应用的主要问题是原数据 的采集, 该次研究中的地形数据为人工野外测量, 研究规模受到一定限制。

关键词 坡度 坡向 DEM 神农架

\section{THE APPLICATION OF DIGITAL ELEVATION MODELS IN COMMUNITY BIODIVERSITY RESEARCH WITH AN EXAMPLE FROM A FAGUS ENGLERIANA-CYCLOBALANOPSIS OXYODON COMMUNITY IN SHENNONGJIA AREA}

\author{
ZHANG Mi ${ }^{1,2}$ XIONG Gao-Ming ${ }^{1} \quad$ CHEN Zhi-Gang ${ }^{1} \quad$ FAN Da-Yong ${ }^{1}$ and XIE Zong-Qiang ${ }^{*}$ * \\ (1 Laboratory of Quantitative Vegetation Ecology, Institute of Botany, Chinese Academy of Sciences, Beijing 100093, China) \\ (2 Graduate School of Chinese Academy of Sciences, Beijing 100039, China)
}

\begin{abstract}
Topography is a primary factor that creates microenvironmental heterogeneity in a landscape. In previous studies, topographic factors, such as slope and aspect, were measured as discrete data and had limited use in analyses. In this paper, a digital elevation model (DEM) was used to analyze the influence of topographic heterogeneity on the distribution patterns of woody plants at the community level by calculating digital topographic values and creating a digital description of the landform.

The establishment of a DEM can be generalized in three steps: 1) gathering the topographic data; 2) data adjustment $\&$ interpolation; and 3 ) displaying the data. To illustrate this process, a DEM was built based on a $0.96 \mathrm{hm}^{2}$ permanent forest plot in the Shennongjia area $\left(31^{\circ} 19^{\prime} 4^{\prime \prime} \mathrm{N}, 110^{\circ} 29^{\prime} 44^{\prime \prime} \mathrm{E}\right)$. The forest was dominated by Fagus engleriana and Cyclobalanopsis oxyodon. Topographic data were gathered when the plot was established. A total of 425 sample points were mapped by elevation. An inverse distance weighted method was used to interpolate data between points. Using Arcview GIS software, the slope and aspect of all 425 points within the plot were calculated. The slope varied from 18.2 to 74.6 degrees in the $0.96 \mathrm{hm}^{2}$ plot. The precision of the DEM was $0.4 \mathrm{~m}$. Using the DEM, the slope and aspect of every point within the permanent plot were calculated and these topographic factors were then used to analyze species distribution patterns. Twentyfour species were chosen to calculate their correlations with slope, aspect and position. The positive and negative correlations with the slope, aspect and position were $7,7,6,3$, and 8,5 respectively.

One problem of using a DEM at small scales is related to the difficulties associated with the collection of the original data. Under the forest canopy, it was difficult to gather topographic data using GPS, remote pictures or other automated tools. To achieve the required precision, all topographic data were acquired using a compass, which limited the scale at which the analysis could be conducted. Although improvements are needed in this method, the DEM provided a lot of information and was a convenient method for analyzing species dis-
\end{abstract}


tributions in relation to topographic factors. Because remote sensing cannot provide a detailed description of species distributions within forest communities, such research should be combined with the establishment of permanent plots.

Key words Slope, Aspect, DEM, Species distribution, Shennongjia

数字高程模型 (Digital elevation model, DEM) 是 地形摸拟及分析的重要工具, 它通过地表点集的空 间坐标 $(\mathrm{x}, \mathrm{y}, \mathrm{z})$ 及其属性数据表示地形表面特征, 是构建地理信息系统的核心部分(李志林,2000)。 在生物学研究中, DEM 大多应用于森林生产力预测 及野生动物栖息地质量评价等方面 (郭志华等, 1999), 本文将其引入小尺度的生物多样性研究, 以 神农架地区的常绿落叶阔叶混交林的研究为例, 介 绍 DEM 建立的基本方法。

地形是环境异质性产生的重要来源, 影响着植 被的分布及群落的组成(方精云等, 1999; 沈泽昊和 张新时, 2000; 沈泽吴和方精云 2001; 宋永昌, 2001)。地形因素对生物多样性的影响正受到越来 越广泛的关注, 对于这一问题的研究要求研究方法 能够反映地形的细微变化, 并能确定这些连续变化 因素的值。数字地形模型可以模拟连续的地形表 面, 量化地形因子, 使之与植被及其它生态因素相结 合, 是群落尺度下研究地形因素对植物种群分布影 响的理想工具。

\section{1 研究地点概况}

研究地点位于湖北省西部的神农架地区 $\left(31^{\circ} 19^{\prime} 4^{\prime \prime} \mathrm{N}, 110^{\circ} 29^{\prime} 44^{\prime \prime} \mathrm{E}\right.$, 海拔 $\left.1750 \mathrm{~m}\right)$, 该地区处 于我国中亚热带与北亚热带过渡地带, 根据中国科 学院神农架生物多样性定位研究站气象资料(海拔 $1290 \mathrm{~m}$ ), 年平均气温为 $10.6{ }^{\circ} \mathrm{C}$, 年降水量为 $1306.2 \sim 1722.0 \mathrm{~mm}$ (1996 2001 年)。同时它也 是我国中部山地与东部丘陵低山区的过渡地带, 在 地质构造上属大巴山山脉褶皱带, 南坡较为平缓, 北 坡较为陡峭, 地貌上具有山高、坡陡、谷深等特点 (中 国生物多样性国情研究报告编写组, 1998)。该地区 山地植被垂直带谱完整, 由低海拔到高海拔依次分布 有常绿榈叶林、常绿落叶阔叶混交林、落叶阔叶林、针 阔混交林、针叶林及杜鹃箭竹灌从( 陈灵芝和王祖望, 1999)。本文涉及的米心水青冈( Fagus engieriana)-曼 青冈( Cyclobalanopsis oxyodon) 常绿落叶阔叶混交群落 是神农架地区一种重要山地植被类型。

研究样地为依照中国科学院森林生物多样性监 测规范设立的永久性固定样地, 面积 $9600 \mathrm{~m}^{2}$ 。群
落调查于 2001 年完成, 群落中共有乔木 46 种, 分属 22 科 27 属, 优势树种为米心水青冈及曼青冈。

\section{2 数字高程模型 (DEM)的建立}

DEM 建立的过程包括: 原始数据的获取、数据 插值、存它、模型输出以及派生数据提取等几个部分 (图 1)。

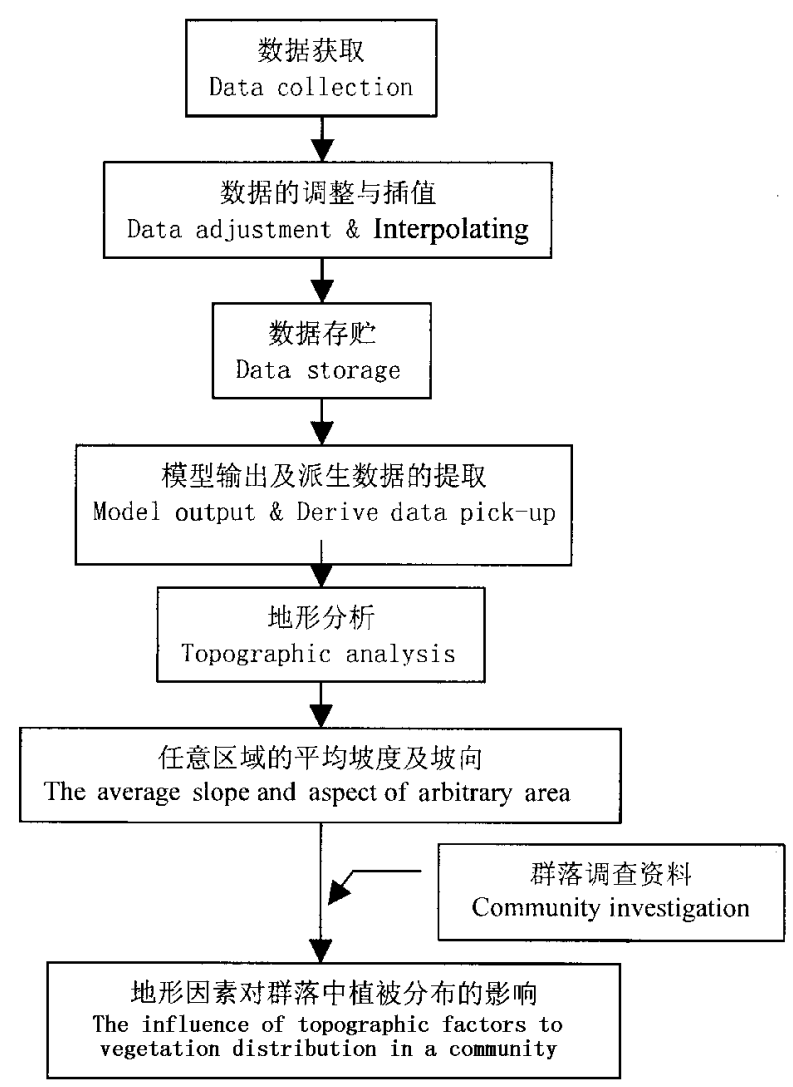

图 1 DEM 建立的过程示意图

Fig. 1 Sketch map of building DEM

\subsection{DEM 数据获取}

DEM 数据获取方法可以分为地面直接测量、摄 影测量及从现有地形图采集等方法。本次研究在神 农架林区进行, 为了保证采样质量, 地形数据采用人 工野外测量的方法获得。小尺度下地形数据必须与 植被调查数据相结合才能达到分析的目的, 本文的 地形资料结合 $0.96 \mathrm{hm}^{2}$ 固定样地的建立采集, 样地 划分为 384 个 $5 \mathrm{~m} \times 5 \mathrm{~m}$ 的小样方, 以样方的四角 作为采样点, 用 DQL-1 型森林罗盘仪 (带三角架及望 
远镜)测得各样点的相对位置及高程, 采样点共 425 个。

\section{2 数据的调整与插值}

真实的地表是连续的, 我们无法对所有点进行 定位, 因此可以使用少量特征点利用数学插值的方 法得到连续表面。插值的方法是根据一组已知的数 据点,构造函数使已知的数据点全部通过该函数。 插值的一般方法包括: 加权法、趋势面法及克立金法 (DeMers, 2001)。

本次研究使用反距离加权法 (Inverse distance weighted, IDW)进行表面插值, 反距离加权法是最常 用的空间内插方法之一，它基于邻近的区域比距离 远的区域更相似这一基本假设,计算简便,适用性也 较广(李新等,2000)。计算公式为:

$$
Z=\frac{\sum_{i=1}^{n} \frac{1}{\left(D_{i}\right)^{p}} Z i}{\sum_{i=1}^{n} \frac{1}{\left(D_{i}\right)^{p}}}
$$

式中, $Z$ 为估计值, $Z_{i}$ 为第 $i$ 个样本 $(i=1, \ldots, n)$, $D_{i}$ 为距离, $p$ 为距离的幂。

\section{DEM 的地形分析}

地形分析是 DEM 的基本功能,利用 DEM 软件 可以自动提取地形因子, 如坡度、坡向、高程变异、地 表粗粘度、曲面面积、体积、谷脊特征分析等。这里 主要是坡度、坡向的提取。

\section{1 坡度、坡向的计算}

地面上某一点的坡度是表示地表面在该点倾斜 程度的一个量。因此, 它是一个既有大小又有方向 的量。自 DEM 理论形成以来, 人们就对计算坡度的 方法进行了大量的研究和试验。迄今为止, 其计算 方法可归纳为 5 种: 四块法、空间矢量分析法、拟合 平面法、拟合曲面法、直接解法。其中拟合曲面法是 求解坡度的最佳方法(李志林, 2000)。拟合曲面法 一般采用二次曲面, 即 $3 \times 3$ 的窗口, 每个窗口中心 为一个高程点。坡度、坡向的定义公式为 (Dozier \& Strahler, 1983):

$$
\begin{aligned}
& \text { Slope }=\tan \sqrt{\text { Slope }_{\mathrm{we}}^{2}+\text { Slope }_{\mathrm{sn}}^{2}} \\
& \text { Aspect }=\text { Slope }_{\mathrm{sn}} / \text { Slope }_{\mathrm{we}}
\end{aligned}
$$

式中, Slope 为坡度, Aspect 为坡向, Slope $e_{\mathrm{we}}$ 为 $\mathrm{X}$ 方向 上的坡度, Slope sn 为 $\mathrm{Y}$ 方向上的坡度。

3.2 米心水青冈-曼青冈群落的地形特征

在对湖北省神农架地区的米心水青冈及曼青冈 组成的常绿落叶阔叶混交林的研究中, 应用上述方
法建立了 $0.96 \mathrm{hm}^{2}$ 样地的地形模型, 并分析群落中 24 种乔木树种对地形因子的适应性。地形数据的 采集结合固定样地的建立完成, 以 384 个 $5 \mathrm{~m} \times 5 \mathrm{~m}$ 样方的四角作为采样点, 用 DQL-1 型森林罗盘仪测 得各样点的相对位置及高程 ( $x, y, z$ ), 共采样 425 个。在地形调查数据的基础上应用 Arcview 软件对 样地的地形数据进行基本的提取。

样地的总坡向为西偏北 $30^{\circ}$, 以半阴半阳坡为 主。以样地总高差及投影边长计算样地总坡度为 $40.01^{\circ}$ 。利用 DEM 计算各样方的平均坡度为 $48.7^{\circ}$ ( $V=87.57, t=6.265, t_{0.01}=2.358, n=123$ ), 样地中 最大坡度为 $74.6^{\circ}$, 最小坡度为 $18.2^{\circ}$ 。图 2 为用 $\mathrm{S}-$ Plus 软件生成的地形三维图, 与等高线图 (图 3) 相 比它更为直接的说明了研究地点的地形变化。样地 随山势起伏, 上部靠近山顶地势较为平缓,下部陡

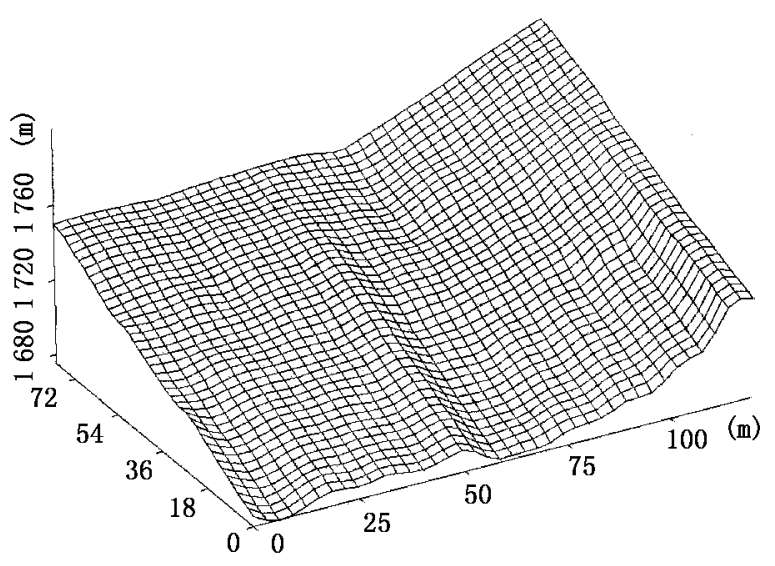

图 $20.96 \mathrm{hm}^{2}$ 固定样地三维地形图 Fig.2 3D map of the $0.96 \mathrm{hm}^{2}$ permanent plot

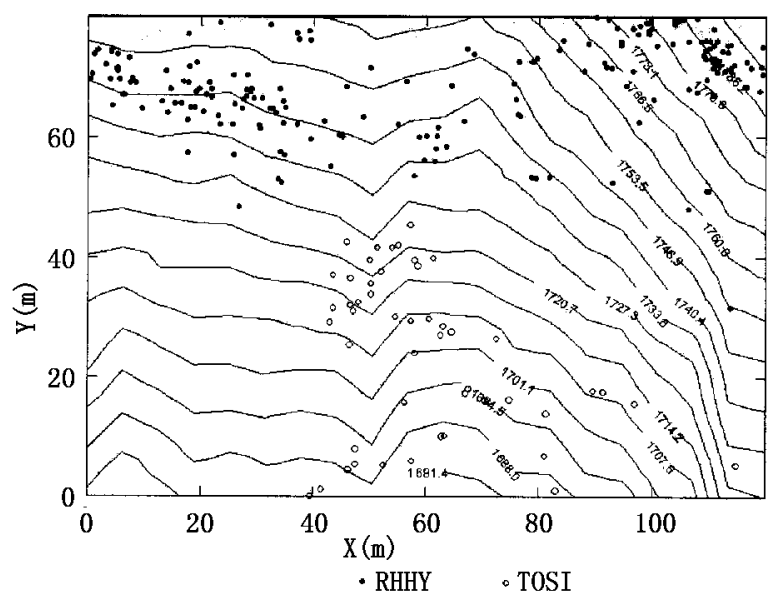

图 $30.96 \mathrm{hm}^{2}$ 固定样地等高线图

Fig. 3 Contour map of the $0.96 \mathrm{hm}^{2}$ permanent plot RHHY: 粉白杜鹃 Rhododendron hypoglaucum TOSI: 香椿 Toona sinensis 
峭, 部分样地处于山坳中。至此, 利用 DEM 模型获 得了 $0.96 \mathrm{hm}^{2}$ 样地地形数据的量化值, $5 \mathrm{~m} \times 5 \mathrm{~m}$ 样方的坡度和坡向值可以作为一个因子计算与植被 分布的关系, 分析地形因素对群落内植被分布的影 响。

\section{3 群落中主要乔木树种对地形的适应性}

地形模型的建立量化了坡度、坡向等地形因子， 使之可以应用于统计分析。统计群落中米心水青冈 及曼青冈等 24 种乔木在不同坡度、坡向及坡位的密 度,并分别与坡度、坡向及坡位因子进行相关分析。 结果表明各物种对不同地形的反应不同,与坡度正 相关的种有米心水青冈、扇叶槭 (Acer flabellatum)、 香椿 ( Toona sinensis)、小叶青皮槭 ( Acer cappadocicum)、鄂椴( Tilia oliveri)、巴东栋( Quercus engleriana) 及鸡爪槭 (Acer palmatum), 说明这些种在坡度较大 的区域也能生长。与坡度呈负相关的种有三桠乌药 (Lindera obtusiloba)、水榆花楸 (Sorbus alnifolia)、山白 树 (Sinowilsonia henryi)、雉栗 (Castanea henryi)、石灰 花楸 (Sorbus folgneri)、粉白杜鹃 (Rhododendron hypoglaucum) 及灯台 (Cornus controversa), 这些种更倾向 于生长在坡度较缓的区域。与坡向呈正相关的有香 椿、梾木 (Cornus macrophylla)、化香 ( Platycarya strobilacea)、曼青冈、石栋( Lithocarpus glaber) 及巴东栋, 说 明这些种倾向于出现在西南坡。与坡向呈负相关的 有粉白杜鹃、紫茎 (Stewartia sinensis)、锥栗, 说明这 些种倾向于出现在西北坡。与坡位呈正相关的是鄂 椴、领春木 (Euptelea pleiosperma)、香椿、华榛( Corylus chinensis)、巴东栎、化香、灯台及扇叶槭, 说明这些种 多出现在样地的下部。与坡位呈现负相关的有四照 花 (Cornus kousa var. chinensis)、粉白杜鹃、三桠乌 药、水榆花楸及石灰花楸,这些种倾向于出现在样地 的上部。详细结果见神农架米心水青冈-曼青冈群 落的地形异质性及其生态影响一文(张䜨等, 2004)。

\section{4 讨 论}

地形作为一种异质性因素显然影响到群落中种 群的分布格局, 它直接影响着植被的立地条件、种子 传播、幼苗及幼树的建成, 进而影响整个群落的结 构、格局及动态, 并为群落中的多物种共存提供了更 多的可能性。本次研究中, DEM 提供了以样方为基 础的坡度、坡向值,这为数量化研究地形因素与植被 分布的关系提供了基础数据。以往的研究中地形因 素只是一个粗略值,一块样地的坡度及坡向往往以 其平均值代表, 无法分析地形的细微变化对植物分
表 1 群落中主要树种分布与地形因素的相关系数

Table 1 The correlation coefficients between the species distribution in the community and the topographic factors

\begin{tabular}{|c|c|c|c|}
\hline \multirow{3}{*}{$\begin{array}{l}\text { 植物名 } \\
\text { Species }\end{array}$} & \multicolumn{3}{|c|}{ 相关系数 Correlation coefficients } \\
\hline & 坡度 & 坡向 & 坡位 \\
\hline & Slope & Aspect & Position \\
\hline 曼青冈(1) & -0.535 & 0.913 & -0.771 \\
\hline 米心水青冈(2) & 0.994 & 0.004 & -0.327 \\
\hline 粉白杜鹃(3) & -0.887 & -0.977 & -0.870 \\
\hline 石栋(4) & -0.800 & 0.895 & 0.655 \\
\hline 四照花（5） & -0.001 & 0.270 & -0.993 \\
\hline 香椿(6) & 0.797 & 0.975 & 0.721 \\
\hline 小叶青皮槭(7) & 0.871 & -0.031 & 0.415 \\
\hline 鄂椴（8） & 0.846 & -0.416 & 0.993 \\
\hline 血皮槭(9） & -0.152 & -0.523 & -0.240 \\
\hline 三桠乌药 (10) & -0.954 & -0.609 & -0.866 \\
\hline 水榆花楸 ( 11) & -0.970 & 0.731 & -0.866 \\
\hline 山白树 (12) & -0.909 & -0.123 & -0.327 \\
\hline 梾木 (13) & -0.078 & 0.961 & 0.655 \\
\hline 稠李( 14) & -0.401 & 0.429 & 0.397 \\
\hline 华榛(15) & -0.014 & 0.247 & 0.866 \\
\hline 巴东栋 (16) & 0.841 & 0.814 & 0.866 \\
\hline 领春木 (17) & 0.592 & 0.263 & 0.952 \\
\hline 雉栗( 18） & -0.943 & -0.813 & -0.866 \\
\hline 化香( 19) & 0.179 & 0.993 & 0.866 \\
\hline 灯台（20） & -0.815 & 0.786 & 0.866 \\
\hline 紫茎(21) & 0.027 & -0.969 & -1.000 \\
\hline 石灰花楸 (22) & -0.901 & 0.000 & -0.933 \\
\hline 鸡爪槭（23） & 0.757 & 0.731 & -0.277 \\
\hline 扇叶槭(24) & 0.981 & -0.555 & 0.866 \\
\hline
\end{tabular}

(1) Cyclobalanopsis oxyodon (2) Fagus engleriana (3) Rhododendron hypoglaucum (4) Lithocarpus glaber (5) Cornus kousa var. chinensis (6) Toona sinensis (7) Acer cappadocicum (8) Tilia oliveri (9) Acer griseum (10) Lindera obtusiloba (11) Sorbus alnifolia (12) Sinowilsonia henryi (13) Cornus macrophylla (14) Prunus padus (15) Corylus chinensis (16) Quercus engleriana (17) Euptelea pleiospermum

(18) Castanea henryi (19) Platycarya strobilacea (20) Cornus controversa (21) Stewartia sinensis (22) Sorbus folgneri (23) Acer palmatum (24) Acer flabellatum

布格局的影响, 而数字高程模型的引入则为此类研 究提供了极大的便利。一方面 DEM 对地形的模拟 是连续的; 同时 DEM 量化了地形因子, 使地形因子 可以与群落调查的资料相结合应用于统计分析。

虽然 DEM 在地形分析中的优势十分明显, 但原 数据的采集问题制约着它在群落水平研究的应用。 原则上研究的尺度越小对于精度要求越高。现有的 地形数据自动采集设备, 如 GPS、全站仪等, 在林下 很难达到测量所需的精度, 部分数据不得不人工采 集, 因此工作量较大, 研究规模受到一定限制。模型 的检验也是一个值得注意的问题。本次研究用 153 个加测点对 DEM 导出高程值进行了检验, DEM 插 值误差为 $0.4 \mathrm{~m}$ 。但是在插值基础上产生坡度和坡 向值则难以检验, 原因在于 DEM 得到的坡度、坡向 
值实际上是邻近区域的平均值, Horn（1981）的算法 涉及周围 8 个点的高程值, 同时考虑了坡向的问题, 而实际测量得到的则是某一点的坡度、坡向值, 而且 由于实际测量沿统一的方向进行, 无法同时兼顾坡 向的细微变化, 因此用实测的坡度、坡向值对 DEM 导出值进行检验比较困难。

本次研究应用的是 Arcview 地理信息系统的 3D 及空间分析模块进行表面插值及坡度坡向计算。目 前认为插值精度最高的为格里金法, Arcview 软件中 仅提供反距离权重法, 这是插值方法当中最为简单 的一种, 而且数据的提取部分还是手动的, 这些都有 待于相关软件的进一步开发与完善。另外, 利用遥 感技术 DEM 在大尺度上的提取可以很容易的做到, 但植被的分布则无法由遥感图像上得到精确的判 别, 对群落内的种群组成判断则更加无能为力, 因此 应用 DEM 分析地形对植被分布的影响应与固定样 地的建立相结合。尽管 DEM 应用于群落内的生物 多样性研究存在着一定的局限性, 但它毕竟为研究 地形因子对于植物分布格局的影响提供了切实可行 的方案, 为生物多样性的研究提供了新的工具。

\section{参 考 文 献}

Chen LZ 陈灵芝), Wang ZW (王祖望) (1999). The Impact of Human Alternation on Ecosystem Diversity (人类活动对生态系 统多样性的影响). Zhejiang Science and Technology Press, Hangzhou, 139 - 197. (in Chinese)

DeMers MN (Translated by Wu FD (武法东), Fu ZT (付宗堂), Wang XN (王小牛)) (2001). Fundamentals of Geographic Information Systems (in English). Electronic Industry Press, Beijing, $22-196$.

Dozier J, Strahler AH (1983). Ground investigations in support of remote sensing. In: Colwell RH ed. Manual of Remote Sensing. Cambridge University Press, Cambridge, 1232.
Editorial Group for Country Studies on Biodiversity in China (中国 生物多样性国情研究报告编写组) (1998). The Nation Report of China Biodiversity Status (中国生物多样性国情研究报 告). China Environment Science Press, Beijing, 1-10. (in Chinese)

Fang JY (方精云)，Guo QH (郭庆华)，Liu GH (刘国华) (1999). Distribution patterns of Chinese Beech (Fagus L.) species in relation to topography. Acta Botanica Sinica (植物学 报), 41, 766-774. (in Chinese with English abstract)

Guo ZH(郭志华), Peng SL (彭少麟), Wang BS (王伯荪), Zhang Z (张征) (1999). Estimation of radiation absorption by Guangdong vegetation using GIS and RS. Acta Ecologica Sinica (生态学报), 19, 441 - 446. (in Chinese with English abstract)

Horn BP (1981). Hill shading and the reflectance map. Proceedings of the Institute of Electrical \& Electronic Engineers, 69, 14.

Li X(李新), Cheng GD(程国栋), Lu L(卢玲) (2000). Comparison of spatial interpolation methods. Advance in Earth Sciences (地球科学进展) , 15, 260-265. (in Chinese with English abstract)

Li ZL(李志林) (2000). Digital Elevation Model (数字高程模 型). Science and Technology Topography University Press, Wuhan, 125 - 158. (in Chinese)

Shen ZH (沈泽昊), Fang JY (方精云) (2001). Niche comparison of two Fagus species based on the topographic patterns of their populations. Acta Phyhtoecologica Sinica (植物生态学 报), 25, 392 - 398. (in Chinese with English abstract)

Shen ZH(沈泽吴), Zhang XS(张新时) (2000). The spatial pattern and topographic interpretation of the forest vegetation at Dalaoling region in the three Gorges. Acta Botanica Sinica (植物 学报), 42, 1089-1095. (in Chinese with English abstract)

Song YC(宋永昌) (2001). Vegetation Ecology (植被生态学). East China Normal University Press, Shanghai, 173 - 176. (in Chinese)

Zhang $\mathrm{M}$ (张㨫), Xiong GM(熊高明), Chen ZG(陈志刚), Fan DY(㚞大勇), Xie ZQ(谢宗强) (2004). The topography heterogeneity of Fagus engleriana-Cyclobalanopsis oxyodon community in Shennongjia region. Acta Ecologica Sinica (生态学报), 24, 2686 - 2692. (in Chinese with English abstract)

责任编委: 傅伯杰 责任编辑: 姜联合 